Advances in Physics

Vol. 00, No. 00, Month-Month 200x, 1-25

\title{
What makes a crystal supersolid?
}

Nikolay Prokof'ev, Department of Physics, University of Massachusetts, Amherst, MA 01003, USA

(Received 00 Month 200x; In final form 00 Month 200x)

For nearly half a century the supersolid phase of matter has remained mysterious, not only eluding - experimental observation, but also generating a great deal of controversy among theorists. Recent

I discovery of what is interpreted as a non-classical moment of inertia at low temperature in solid

${ }^{4} \mathrm{He}[1-4]$ has elicited much excitement as a possible first observation of a supersolid phase. In the Ztwo years following the discovery, however, more puzzles than answers have been provided to the fundamental issue of whether the supersolid phase exists, in helium or any other naturally occurring condensed matter system. Presently, there is no established theoretical framework to understand the body of experimental data on ${ }^{4} \mathrm{He}$. Different microscopic mechanisms that have been suggested to -underlie superfluidity in a perfect quantum crystal do not seem viable for ${ }^{4} \mathrm{He}$, for which a wealth I of experimental and theoretical evidence points to an insulating crystalline ground state. This perSpective addresses some of the outstanding problems with the interpretation of recent experimental Dobservations of the apparent superfluid response in ${ }^{4} \mathrm{He}$ (seen now by several groups [5-7]) and Odiscusses various scenarios alternative to the homogeneous supersolid phase, such as superfluidity Sinduced by extended defects of the crystalline structure which include grain boundaries, dislocations, anisotropic stresses, etc. Can a metastable superfluid "glassy" phase exist, and can it be relevant to some of the experimental observations? One of the most interesting and unsolved fundamental ques-

tions is what interatomic potentials, given the freedom to design one, can support an ideal supersolid $>$ phase in continuous space, and can they be found in Nature.

\section{Introduction}

The textbook notion of a perfect crystal at $T=0$, is that of a periodic array of unit cells, all comprising the same integer number of particles, $\nu=\int_{\Omega} d \boldsymbol{r} \rho(\boldsymbol{r})$, where $\Omega$ is the unit cell volume and $\rho(\boldsymbol{r})$ is the average particle density profile. For simplicity, let us consider a single component crystal similar to ${ }^{4} \mathrm{He}$. We assume that the elementary constituents (atoms or molecules) can be regarded as structureless particles; furthermore, we assume that they obey Bose Statistics.

$\because$ The supersolid phase (SFS) can be generally defined as one that combines crystalline properties, such as shear modulus and broken translation symmetry, with frictionless mass transport through the solid bulk. The striking, simultan'eous presence of solid and superfluid properties in the same condensed matter "system, will result in a number of phenomena that defy our everyday experience. One such phenomenon is schematically depicted in Fig. 1, showing a SFS 

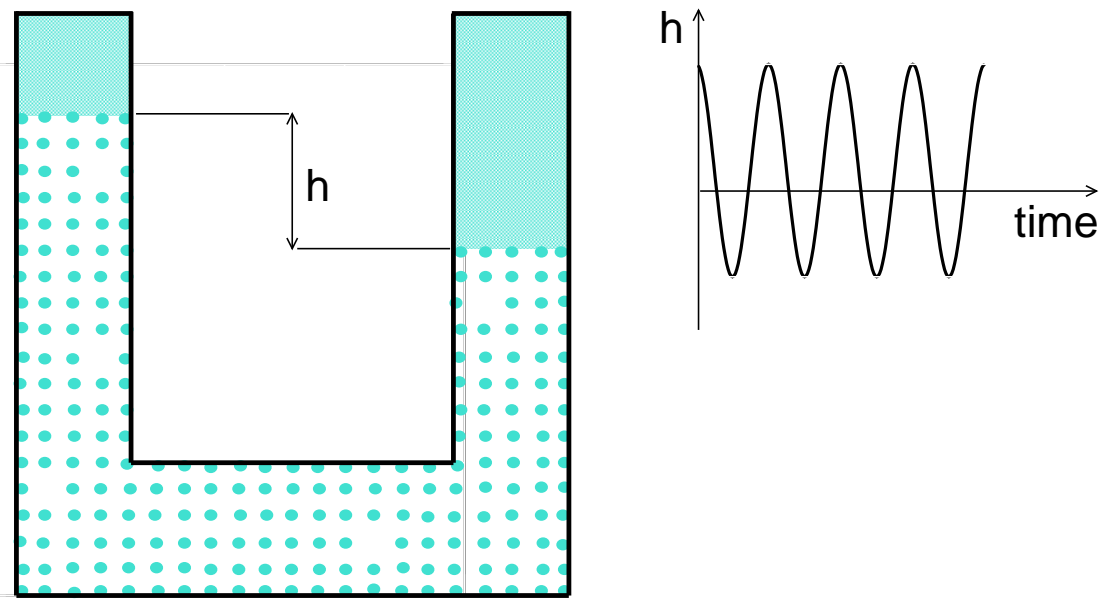

Figure 1. Supersolid crystal (represented by periodic arrays of dots) in coexistence with the superfluid liquid of lower density (shaded regions on top of the U-shaped closed tube) in the gravity field. If initial conditions are such that supersolid levels to the left and to the right are at different height, the system will start oscillating by melting on one side and recrystallizing released density on another side with coherent dissipationless mass flow through the otherwise static crystalline structure. [The assumption is that the flow velocity remains below the critical value, or, $h$ is small enough, and the supersolid-liquid interface is rough.]

sample (region with periodic arrays of dots) placed inside a U-shaped vessel, in coexistence with the superfluid liquid. At $T=0$ undamped oscillations of the solid levels in the two sections of the U-shape vessel will be observed, as long as the maximum flow velocity remains below the critical value and the liquid-solid interface is rough.

Another landmark of SFS behavior (see, for instance, Ref. [8]), is the reduction of the moment of inertia of a solid sample with respect to its classical value (commonly referred to as Non-Classical Rotational Inertia, or NCRI)

$$
I(T)=I_{\text {class }}\left(1-n_{s}(T) / n\right) .
$$

Here $n_{s}(T)$ is the superfluid density, and $n$ is the particle density in the system. The NCRI effect can be observed by enclosing a known amount of solid in a vessel, which is then set in rotation about its axis. The moment of inertia of the system is related to the (measurable) resonant period of oscillation of the rotating system. A drop of the moment of inertia at low temperature, is interpreted as decoupling of part of the solid (the superfluid fraction) from the rotation.

The history of ideas on how superfluidity can occur in a crystalline solid is 


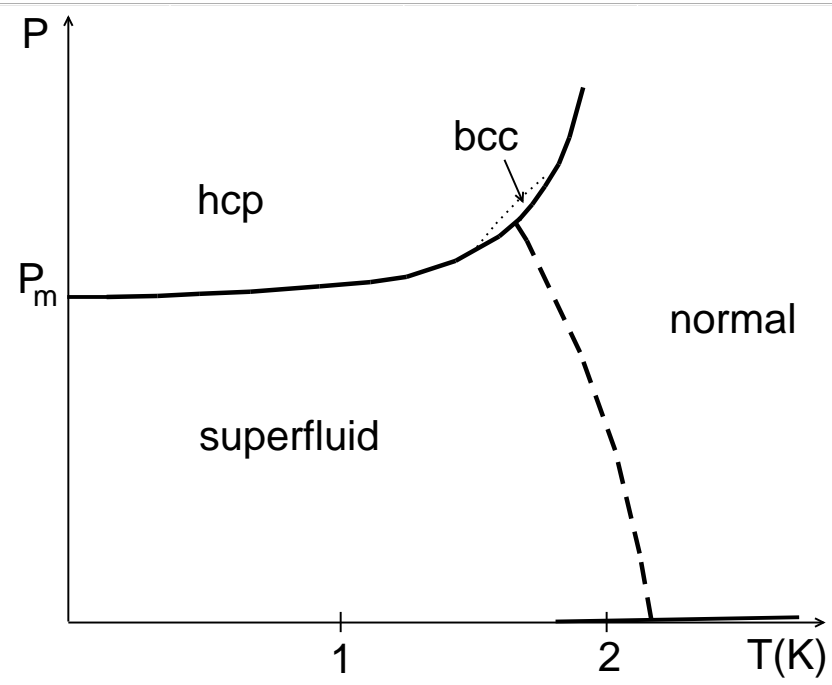

Figure 2. A sketch of the ${ }^{4} \mathrm{He}$ phase diagram. At low temperature the helium superfluid undergoes a weak first-order phase transition to the hexagonal close-packed (hcp) solid phase at pressure $P_{m} \approx 25.6$ bar.

quite old. On very general grounds, one expects that the "textbook" crystal ought to be insulating, i.e., with vanishing superfluid density $n_{s}$, and offdiagonal correlations decaying exponentially in space. Penrose and Onsager (PO) were the first to argue in favor of this point of view $[9,10]$, starting from the picture of atoms localized around their equilibrium lattice positions. However, their argument has never been regarded as the final word in the discussion of whether the superfluid solid phase of matter is possible. C. N. Yang made a comment that long-range phase correlations in the solid state could occur in systems characterized by a high degree of atomic delocalization [11]. Helium is unique in this regard; quantum properties of light helium atoms in combination with the relatively weak interatomic potential (the well depth is only $\sim 10 \mathrm{~K}$ ) prevent them from making a crystal at zero pressure, see Fig. 2. An objection to the PO treatment was that regarding atoms as being localized around lattice points underestimates the role of exchange processes, which may allow for the (perhaps remote) possibility of superflow, even with an integer $\nu[8,12]$.

Another proposal for the supersolid phase has its origin in the observation that there is no fundamental reason why crystals should necessarily be commensurate, i.e., feature an integer number of particles per unit cell, on average. A dilute gas of vacancies, or interstitials, which occur in all solids at finite temperature and ought to be highly mobile in a helium crystal, could be also present in the ground state giving rise to Bose-Einstein condensation 
and superfluidity at low temperature $[13,14]$.

Though certainly plausible, most theoretical scenarios leading to the SFS phase are phenomenological and qualitative when it comes to making predictions for a particular Hamiltonian; they have never been supported by robust microscopic calculations, including all quantum-mechanical effects and based on realistic models of crystals. ${ }^{1}$ Still, their appeal is so strong that they inspired decades of active research and continue to do so.

Remarkable manifestations of quantum behavior on a macroscopic scale in supersolids are expected to render the observation of the SFS state rather unambiguous, as long as it occurs under conditions (e.g., temperature) accessible to the experiment. Such an observation has eluded experiments for some thirty years (for a review of the experimental evidence prior to 2004, see, for instance, Ref. [17]); it was only in 2004 that the first convincing evidence of a SFS phase of ${ }^{4} \mathrm{He}$ was published. The phenomenon was first reported for a solid embedded in a porous Vycor matrix by [1] Kim and Chan (KC); shortly thereafter, it was extended to the system embedded in a matrix of porous gold (with a characteristic pore size two orders of magnitude greater than in Vycor), and eventually to the bulk crystal [2]. In all cases, the observed NCRI at low temperature was identified as the onset of supersolidity in ${ }^{4} \mathrm{He}$. It should be emphasized that the supersolid interpretation of the data is supported by the velocity/amplitude dependence of the NCRI signal and the crucial test that the NCRI effect goes away (is suppressed by two orders of magnitude) when the flow channel around the rotation axis is blocked. These results constitute a major problem for the non-superfluid kinetic interpretation.

The Kim and Chan discovery sparked a renewed effort in the investigation of the SFS phase of matter. At the time of this writing, it seems fair to state that things have turned out to be considerably more complicated (and thus more interesting) than expected. As the initial experiment by Kim and Chan is being repeated by other groups, and concurrently different experiments and reliable microscopic (first-principles) calculations, based on state-of-the-art computational techniques are being carried out, consensus is building that the early microscopic scenarios of supersolidity, described above, are not viable to explain the body of experimental data. For example, there is now strong theoretical and experimental evidence that ${ }^{4} \mathrm{He}$ is a commensurate crystal, and such crystals made of a single species of particles obeying Bose statistics (e.g., ${ }^{4} \mathrm{He}$ atoms) are always insulating (more precisely, the commensurate SFS phase

\footnotetext{
${ }^{1} \mathrm{~A}$ major current area of investigation, in the broader context of the study of exotic phases of matter, focuses on quantum lattice models. Supersolid phases have been predicted to occur in some of these models $[15,16]$ and may soon be observed experimentally, e.g. in optical lattices. Albeit of clear fundamental interest, this topic is not part of the discussion in this paper. We also do not discuss multicomponent cases in which one component forms a solid and, thus, acts as an external periodic potential for the other. Such cases are expected to behave similarly to lattice models.
} 
has zero probability of being observed). Measurements and calculations of vacancy and interstitial properties indicate that the Andreev-Lifshits-Chester scenario of vacancy-induced supersolidity does not occur in solid helium, even out of equilibrium. Therefore, attention is now shifting to a variety of inhomogeneous scenarios, that may account for many puzzling (and often apparently conflicting) experimental results.

This paper is organized as follows: we shall first review the theoretical framework for supersolidity, including the early suggestions as well as some more recent proposals of microscopic mechanisms. They will be discussed in the light of both early and recent analytical and numerical results. We shall then examine the core of experimental data, which at this time includes observations of NCRI as well as flow experiments [18] and the most recent experiment on grain boundary superfluidity [19], and propose that, while a definitive theoretical explanation of the observed phenomena is not yet available, there are good reasons to look at disordered and/or inhomogeneous scenarios. Specifically, we shall illustrate how the effect of disorder can hold the key to interpreting an important part of the phenomenology. Finally, we shall outline some possible directions of experimental and theoretical investigation.

This perspective is a quick response to the fast developing topic, not a comprehensive review. I apologize if some important contributions to the field were overlooked. Given a broad range of opinions and opposing points of view on the subject, there is certainly a degree of personal bias towards which arguments and results are more convincing and should be given more "weight" in the discussion. The hope is that the story is provocative enough to stimulate additional research efforts to understand the remarkable phenomenon of supersolidity.

\section{Supersolid ground state}

Often the discussion starts with the proposal for the supersolid ground state wave function $\Psi_{G}$ though it is certainly allowed by the theory that in a particular material SFS exists only as a finite-temperature phase. In the PenroseOnsager picture of atoms localized around equilibrium points $\left\{\boldsymbol{R}_{i}\right\}$, the ground state wave function is simply a product of localized Wannier orbitals

$$
\Psi_{G}^{(P O)}=\sqrt{\frac{1}{N !}} \sum_{P} \prod_{j=1}^{N} \varphi\left(\boldsymbol{R}_{j}-\boldsymbol{r}_{P j}\right)
$$


where the sum $\sum_{P}$ is over all permutations $P$ of particle labels. Using the definition of the single-particle density matrix at zero-temperature

$$
n\left(\boldsymbol{r}, \boldsymbol{r}^{\prime}\right)=\int \ldots \int d \boldsymbol{r}_{2} d \boldsymbol{r}_{N} \Psi_{G}\left(\boldsymbol{r}, \boldsymbol{r}_{2}, \ldots, \boldsymbol{r}_{N}\right) \Psi_{G}\left(\boldsymbol{r}^{\prime}, \boldsymbol{r}_{2}, \ldots, \boldsymbol{r}_{N}\right)
$$

and simple properties of the variational state $\Psi_{G}^{(P O)}$ one readily obtains (by substituting $\Psi_{G}^{(P O)}$ for $\Psi_{G}$ above)

$$
n\left(\boldsymbol{r}, \boldsymbol{r}^{\prime}\right)=\frac{1}{N} \sum_{i} \varphi\left(\boldsymbol{R}_{i}-\boldsymbol{r}\right) \varphi\left(\boldsymbol{R}_{i}-\boldsymbol{r}^{\prime}\right)
$$

Since in the $\left|\boldsymbol{r}-\boldsymbol{r}^{\prime}\right| \rightarrow \infty$ limit either $\boldsymbol{r}$ or $\boldsymbol{r}^{\prime}$ has to be far from the equilibrium lattice point $\boldsymbol{R}_{i}$, the density matrix decays to zero exponentially fast for localized Wannier orbitals. This immediately implies no off-diagonal long-range order (ODLRO) and thus no superfluidity in the system.

Here, we adopt the following definition of ODLRO: we say that it is present in the system, if the integral

$$
\int n\left(\boldsymbol{r}, \boldsymbol{r}^{\prime}\right) d \boldsymbol{r} \rightarrow \infty, \quad \text { (Def. ODLRO) }
$$

diverges in the thermodynamic limit. This definition is different and more general than that based on finite condensate density $\left(n_{0}\right)$, which requires that the integral be proportional to the system volume, in the thermodynamic limit $[8,10,20,21]$. It makes perfect sense to define ODLRO in such a way that superfluidity automatically leads to ODLRO and vice versa. In particular, Eq. (5) allows one to talk about ODLRO in two dimensional (2D) systems at finite temperature (often referred to as topological order [22]) for which the condensate density is zero.

In this article, we restrict ourselves to the single-particle superfluidity. In general, one has to look at the many-body density matrix to see if there is ODLRO in some finite-order channel. For example, it is certainly possible that $\mathrm{M}$ atoms form M-molecules and superfluidity happens than at the molecular level - in this case one has to study the M-particle density matrix. Or, molecular hydrogen would be a good example here, by neglecting the internal structure of molecules and treating them as point particles (probably at the expense of introducing complex effective many-body interactions) ODLRO can again be discussed in the single-particle "molecular" density matrix. There is no fundamental difference between the two pictures.

The superfluid density (formally a tensor) is a linear response coefficient which controls the free-energy increase in a system with periodic boundary 
conditions $(\mathrm{BC})$ when these $\mathrm{BC}$ are twisted, i.e. if $L_{\alpha}$ is the linear system size in direction $\alpha=\hat{x}, \hat{y}, \hat{z}$ we require that $\Psi\left(\ldots, r_{\alpha}+L_{\alpha}, \ldots\right)=e^{i \varphi_{\alpha}} \Psi\left(\ldots, r_{\alpha}, \ldots\right)$ (there are other definitions of $n_{s}$, e.g. through the NCRI, Eq. (1), which can be shown to be identical to the one given below)

$$
F(\boldsymbol{\varphi})-F(0)=\sum_{\alpha \alpha^{\prime}} \frac{\left(n_{s}\right)_{\alpha \alpha^{\prime}}}{2 m} \frac{V}{L_{\alpha} L_{\alpha^{\prime}}} \varphi_{\alpha} \varphi_{\alpha^{\prime}} .
$$

Here $V=\prod_{\alpha} L_{\alpha}$ is the system volume. Twisted BC can be avoided by introducing instead a gauge phase with the gradient $\nabla \phi$ and writing the free-energy density (on the largest scales) as

$$
f(\phi)-f(0)=\sum_{\alpha \alpha^{\prime}} \frac{\left(n_{s}\right)_{\alpha \alpha^{\prime}}}{2 m}(\nabla \phi)_{\alpha}(\nabla \phi)_{\alpha^{\prime}} .
$$

Using "connectivity" arguments $[8,20,21]$ one can show that the system is sensitive to twisted BC only if it has ODLRO defined in Eq. (5).

The wave function (2) takes into account zero-point motion and local exchange processes between identical particles (these effects are included in the shape of the Wannier orbitals); nevertheless, it describes an insulating state, as long as $\varphi\left(\boldsymbol{R}_{i}-\boldsymbol{r}\right)$ are localized. By moving away from two major assumptions made in the construction of the insulating ground state (2), one arrives at two, apparently quite different possibilities for the supersolid wave function. If the role of exchange is underestimated in localizing particles around different lattice points, then a better variational state might be of the BEC form

$$
\Psi_{G}^{(B E C)}=\prod_{i=1}^{N}\left(\frac{1}{\sqrt{N}} \sum_{j=1}^{N} \varphi\left(\boldsymbol{R}_{j}-\boldsymbol{r}_{i}\right)\right)
$$

with macroscopic condensate in the single-particle state $\frac{1}{\sqrt{N}} \sum_{j=1}^{N} \varphi\left(\boldsymbol{R}_{j}-\boldsymbol{r}\right)$. The BEC state (as well as $\Psi_{G}^{(P O)}$ ) can be further improved by multiplying it by a many-body correlation factor of the Jastrow form, namely $\exp \left\{-\sum_{i<j=1}^{N} u\left(\boldsymbol{r}_{i}-\boldsymbol{r}_{j}\right)\right\}$, whose purpose is to account for the strong repulsive core of the interatomic potential, and suppress the condensate fraction $\left(n_{0}\right)$ to a value much smaller than unity $\left(n_{0}\right.$ is already below $10 \%$ in the superfluid ${ }^{4} \mathrm{He}$ at zero pressure [23-25]). By construction, the Jastrow factor ought not alter, nor eliminate the underlying lattice structure.

Equation (7) offers an alternative view of the supersolid state, namely that of a superfluid phase with a density wave modulation. However, it suffers from a fundamental shortcoming, in that the number of lattice points $N_{L}$ is arbi- 
trarily assumed to be the same as the number of particles. In a superfluid the density-wave parameters of any variational ansatz are independent thermodynamic variables, to be selected through energy minimization. At this point, we recall that superfluidity and ODLRO imply that part of the system matter is characterized by a classical field component, for which the notion of particle number can be safely ignored without loss of generality. [This component represents low-momentum states with large occupation numbers.] Thus, the condition $N_{L}=N \times$ integer can be satisfied only by accident, which is to say that a commensurate supersolid in continuous space has a probability of zero measure to be found in Nature. As a result, Eq. (7) can not describe the generic ground state of a realistic system [26]. In order to illustrate more vividly how ODLRO relates to the presence of gapless vacancies and/or interstitials and incommensurability of a single component solid in continuous space, consider the simple example of a large-amplitude (classical) standing wave of the electro-magnetic field in the typical optical table experiment. In this case, the question would never be asked whether the number of photons per wave period is an integer.

Another possible modification of Eq. (2), one that retains the picture of localized orbitals, consists of allowing the number of particles $N$ to be less than the number of lattice sites $N_{L}$, i.e., assuming that the ground state has no energy gap for the creation of vacancies ${ }^{1}$

$$
\Psi_{G}^{(A L)}=\sqrt{\frac{\left(N_{L}-N\right) !}{N_{L} !}} \sum_{\left\{k_{1} \ldots k_{N}\right\}} \prod_{j=1}^{N} \varphi\left(\boldsymbol{R}_{k_{j}}-\boldsymbol{r}_{j}\right),
$$

where the the sum is over all possible sets of $N$ lattice points out of $N_{L}$ available. Now, the outcome of the calculation for the density matrix is quite different because in the sum over $i, j$

$$
n\left(\boldsymbol{r}, \boldsymbol{r}^{\prime}\right)=\frac{N_{L}-N}{N_{L}^{2}} \sum_{i, j=1}^{N_{L}} \varphi\left(\boldsymbol{R}_{i}-\boldsymbol{r}\right) \varphi\left(\boldsymbol{R}_{j}-\boldsymbol{r}^{\prime}\right) .
$$

one can always find terms with $\boldsymbol{R}_{i}$ and $\boldsymbol{R}_{j}$ close, respectively, to $\boldsymbol{r}$ and $\boldsymbol{r}^{\prime}$, no matter how large their separation. If, in the spirit of a tight-binding approach, we move from a description in terms of continuous space variable $\boldsymbol{r}$ and $\boldsymbol{r}^{\prime}$, to that of lattice site positions for the particle, by introducing site creation (and, analogously, annihilation) operators $b_{l}^{\dagger}=\int d \boldsymbol{r} \varphi\left(\boldsymbol{R}_{l}-\boldsymbol{r}\right) b_{\boldsymbol{r}}^{\dagger}$, then Eq. (9)

\footnotetext{
${ }^{1}$ The same argument can be used for interstitials, although typically their energy cost is higher than for vacancies; henceforth, we shall implicitly make this assumption consistently throughout the paper
} 
can be recast in the following form (taking lattice translation invariance into account)

$$
n(l)=\sum_{k} n(k, k+l)=\sum_{k}\left\langle b_{k}^{\dagger} b_{k+l}\right\rangle=\frac{N_{L}-N}{N_{L}} \equiv n_{v} .
$$

The final result is consistent with the picture of BEC of a non-interacting vacancy gas, with dimensionless lattice concentration $n_{v}$, introduced by Andreev and Lifshitz [13]. One has to assume next that the effective interaction between gapless vacancies is repulsive, in order to ensure system stability (i.e., to prevent the vacancy gas from collapsing).

As was noted by Reatto [27] and Chester [14], it is not at all necessary to break translational invariance explicitly in the SFS state with vacancies, by specifying the set of equilibrium particle positions $\left\{\boldsymbol{R}_{i}\right\}$. An analogy between the Jastrow wave function

$$
\Psi_{G}^{(J)} \propto \exp \left\{-\sum_{i<j=1}^{N} u\left(\boldsymbol{r}_{i}-\boldsymbol{r}_{j}\right)\right\}
$$

and the partition function of a classical system of interacting particles with pairwise potential $v^{(c l)}(r)=T u(r)$ at finite temperature, suggests that among (11) there are variational states which feature spontaneous crystalline order, as classical systems are known to crystallize at sufficiently low temperature. States (11) are also superfluid since they feature ODLRO, and incommensurate [27]. Regarding the last property, one may observe that, due to lack of vacancyinterstitial symmetry in continuous solids, the probability of having $N=N_{L}$ is of zero measure [26] (an obvious statement for a classical solid at finite $T$ ).

Based on all of the above considerations, one might be led to thinking that the Jastrow wave function may hold the key to the microscopic understanding of supersolidity. However, there are fundamental problems associated with Eq. (11), chiefly that it can not possibly describe realistic solids of systems with short-range interactions (such as helium), for which the condensate fraction is tiny (less than $10^{-3}$ ). The reasoning supporting this criticism is offered in the next Section.

The most important conclusion of this Section is that only zero-point vacancies or interstitials can make an ideal crystal supersolid. There is no other ideal-crystal scenario for the SFS state though microscopic mechanisms leading to the incommensurate crystalline groundstate might be very involved. However, the story does not end here, since we did not question yet what happens when quantum crystals are not perfect, i.e. contain defects such as dislocations and grain boundaries, or even loose their crystalline order com- 
pletely and form an amorphous solid. It appears, that topological defects and glasses offer an alternative approach to supersolid phenomenon. In what follows we examine which of the two possibilities is most likely to operate in helium.

\section{Insulating $h c p$ crystals of ${ }^{4} \mathrm{He}$.}

Existing theories can not answer the question at what pressures and temperatures one has to look for the SFS phase, or even whether the SFS phase exists at all in a given Hamiltonian, e.g. in helium. Several aspects have emerged as necessary conditions that the SFS state has to satisfy, in order to exist; consequently, any of them can be used to probe hcp crystals of helium theoretically. [At the time of this writing, there is no experimental evidence of structural transitions in solid helium at low temperatures $T<1 K$ and moderate pressures, say $P<200$ bar.] By definition, the SFS state has a finite superfluid density $n_{s}$. Since superfluidity and ODLRO (as defined above) must be present simultaneously, one can also look at asymptotic properties of the single-particle density matrix, which is nothing by the zero time limit $n(\boldsymbol{r})=G(\boldsymbol{r},-0)$ of the Matsubara Green function

$$
G(\boldsymbol{r}, \tau)=V^{-1} \int d \boldsymbol{r}^{\prime}\left\langle\mathcal{T}\left\{\hat{b}_{\mathbf{r}^{\prime}+\mathbf{r}}(\tau) b_{\mathbf{r}^{\prime}}^{\dagger}(0)\right\}\right\rangle
$$

Here $\langle\ldots\rangle$ stands for the thermal expectation value, $\mathcal{T}$ is the time-ordering operator, $-\beta / 2 \leq \tau \leq \beta / 2$, and $b_{\mathbf{r}}^{\dagger}(\tau)$ is the Bose particle creation operator in the Matsubara representation. For $\tau<0(\tau>0)$ one is computing the Green function for a vacancy (interstitial atom). If, over large distances and/or long times, $n(r)$ and $G(\boldsymbol{r}, \tau)$ decay exponentially, then the state is non-superfluid, i.e., insulating. Moreover, if $n(r)$ and $G(\boldsymbol{r}, \tau)$ are temperature-independent up to a certain length/time scale, then one can claim that ground state properties on the corresponding scales are being explored (this is most readily seen in the path-integral framework). Finally, by performing simulations in the grand canonical ensemble one can investigate whether the solid is commensurate or has gapless vacancies/interstitials in the ground state.

All of the above criteria have been used in recent first-principles simulations of $h c p$ helium crystals. They are based on the standard pairwise interatomic potential for helium [28] which essentially did not change in the last decades and is known to capture all important properties of condensed and gaseous helium with the relative accuracy of the order of one percent. The study of exchange cycles [29] reveals that they are extremely rare in the solid phase and indicative of the insulating behavior [30]. One may recall that macroscopic 

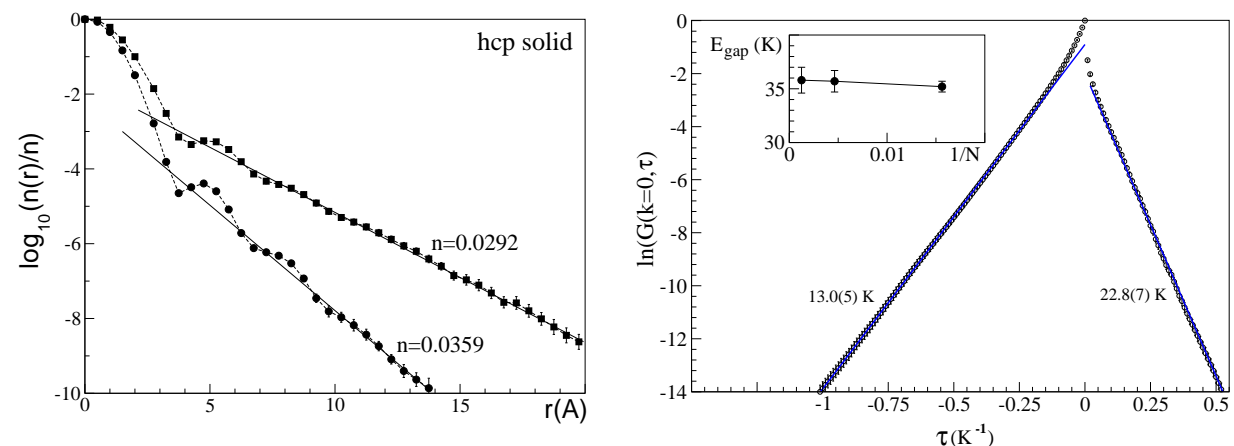

Figure 3. Simulation results for the ideal hcp crystal of $N=800$ atoms at low temperature $T=0.2 \mathrm{~K}$. Left panel [32]: the density matix close to the melting curve, $P=32$ bar $(n=0.0292$ $\left.\mathrm{A}^{\circ-3}\right)$, and at elevated pressure $P=155$ bar $\left(n=0.0359 \mathrm{~A}^{-3}\right)$. The solid line is representing an exponential decay. Right panel [38]: the zero-momentum Green function at the melting density $n_{\circ}=0.0287 \mathrm{~A}^{\circ}$. Symbols refer to numerical data, solid lines are fits to the long-time exponential decay. The given numerical values are the interstitial $\left(\Delta_{\mathrm{I}}=22.8 \pm 0.7 \mathrm{~K}\right)$ and the vacancy $\left(\Delta_{\mathrm{V}}=13.0 \pm 0.5 \mathrm{~K}\right)$ activation energies, inferred from the slopes of $G$. The inset shows the vacancy-interstitial gap $E_{\text {gap }}=\Delta_{\mathrm{I}}+\Delta_{\mathrm{V}}$ for different system sizes.

exchange cycles are necessary for superfluidity, since

$$
n_{s}=\frac{m T\left\langle\boldsymbol{W}^{2}\right\rangle}{d L}
$$

where $m$ is the helium mass, $d$ is the dimensionality, $L$ is the linear system size (periodic boundary conditions are assumed), and $\boldsymbol{W}=\left(W_{x}, W_{y}, W_{z}\right)$ are winding numbers, counting how many times exchange cycles involving many particles wind around the system boundaries [31]. To simplify, we ignore here the tensor structure of $n_{s}$ in the crystal. Initial concerns about ergodicity problems of simulations probing large exchange cycles [30] were eliminated in subsequent path-integral Monte Carlo (PIMC) simulations based on the Worm Algorithm [32], which established that the superfluid fraction $n_{s} / n$ is unobservably small (below $10^{-9}$ ), even in finite-size crystals comprising only 800 atoms.

More detailed evidence for the insulating behavior of helium crystals is provided by computations of the single-particle density matrix and the zeromomentum Matsubara Green function [32,38]. In Fig. 3, we clearly see an exponential decay of the one-body density matrix $n(r)$ at large distances, both in the vicinity of the melting curve (pressure $P=32$ bar) and at the pressure of $P=155$ bar. The observed behavior is independent of temperature for $T<1.5 \mathrm{~K}$, and there is good agreement between independent calculations of $n(r)$ performed by different groups at the melting pressure [33].

From the exponential decay of the zero-momentum $G(\mathbf{k}=0, \tau)$ (left panel 
of Fig. 3), one can obtain an activation energy $\Delta=\Delta_{\mathrm{V}} \approx 13 \mathrm{~K}$ for a vacancy $\left(\Delta_{\mathrm{I}} \approx 23 \mathrm{~K}\right.$ for an interstitial) by studying

$$
G(\mathbf{k}=0, \tau) \propto e^{-|\tau| \Delta}, \quad|\tau| \rightarrow \infty
$$

for $\tau<0(\tau>0)$. We note that these energies are quite large compared to the characteristic temperature of $0.2 \mathrm{~K}$ of the $\mathrm{KC}$ experiment, all but ruling out the possibility that thermally excited vacancies may be behind the supersolid phenomenon (it should also be noted that $\Delta_{V}$ and $\Delta_{I}$ increase with pressure) [38]. This conclusion is consistent with the absence of thermally activated vacancies at low $T$, as inferred from impurity mobility experiments (summarized in Ref. [34]), as well as high-precision studies of the liquid-solid phase diagram [35]. One may note that simulations of gaps in a system comprising 800 atoms are essentially probing the thermodynamic limit, as finite-size effects are negligible (see inset of left panel of Fig. 3).

The analysis of the X-ray data at low temperature in terms of activated vacancies [34] was questioned in Ref. [40]. Specifically, the formula

$$
n_{v}=e^{-\Delta_{V} / T}\left(\frac{m T}{2 \pi}\right)^{3 / 2},
$$

with reported activation energies, in combination with the calculated [36] vacancy effective mass, can not account for the observed change of the lattice constant (the effect would be too small). However, since energies of optical and acoustic phonons at the Brillouin zone boundary are also $\sim 10 \mathrm{~K}$, one can not exclude a conventional explanation in terms of crystal anharmonicity. It seems, that all one can state with relative confidence is that the vacancy concentration is below $1 \%$.

In the most conservative approach, the above-mentioned theoretical results show that commensurate $h c p$ crystals of ${ }^{4} \mathrm{He}$ are insulating and locally stable since single vacancies have a finite energy cost. This does not exclude, strictly speaking, a remote possibility that the true ground state may have a finite, albeit small concentration of vacancies $[39,40]$. Such scenarios, however, are ruled out by strong attractive interaction between vacancies; indeed, when vacancies are forcibly introduced in the crystal at any finite concentration (in a "computer experiment"), they immediately phase separate from the crystal bulk [38]. Under realistic experimental conditions, they will quickly anneal at grain boundaries and dislocations; if the latter are absent, then vacancies will form liquid droplets (at the melting curve) or dislocation loops. In any case, the net result is that the commensurate insulating $h c p$ crystal is the true ground state.

In addition to the original picture of the dilute zero-point vacancy gas 
$[13,14]$, over the past few years there have been several theoretical proposals for the ideal supersolid phase of helium [39,41-44], differing in the microscopic mechanisms of supersolidity. Some of the early works suggesting commensurate SFS phases, were subsequently amended, to respect the theorem that such phases should necessarily feature gapless vacancies (not necessarily in the dilute gas phase). In this respect, Refs. $[41,43]$ are still in error. An interesting idea was proposed in Ref. [45], that $h c p$ crystals may become SFS only under anisotropic stresses, which are likely to occur in the experiment. However, simulation results for $h c p$ crystals at the melting curve, show that vacancy and interstitial gaps hardly change, even under very large anisotropic stress [46]. It appears at this point that phenomenological scenarios and mean-field theories of supersolidity do not work for ${ }^{4} \mathrm{He}$, and one has to seek the explanation of recent experimental results outside the paradigm of the homogeneous $h c p$ crystal.

Before reviewing crucial experimental facts, let us discuss the most important shortcomings (including some unphysical properties) of Jastrow, as well as of the related "shadow" [47] variational wave functions, in the context of helium. Calculations based on these wave functions predict both (i) a very small, but finite, condensate fraction for solid ${ }^{4} \mathrm{He}$ near the melting curve (below $10^{-4}$ ) and (ii) large activation energies for vacancies in solid helium samples comprising several hundred atoms [48]. In the presence of short-range interactions among atoms, the physics of vacancy formation is local in nature. All of the energy contributions quickly approach their thermodynamic limit value, as the system size is increased. Indeed, the kinetic energy of localization in a volume $L^{3}$ is proportional to $1 / L^{2}$ (this contribution is not present in a system with periodic boundary conditions). The direct coupling of the removed atom with the rest of the system converges as $1 / L^{3}$ for the Van der Waals interaction; the same law describes convergence of the deformation energy. The fast disappearance of finite-size effects as $L$ is increased, is observed in essentially all numerical simulations of condensed helium (see, e.g. inset in Fig. 3), including those based on the variational approach. On the other hand, vacancies are gapless in a state described by a Jastrow wave function by construction! It means that if variational calculations were taken to larger system sizes they would have predicted negative corrections to the vacancy energy which increase, not decrease, with $L$, making $\Delta_{I}$ negative for $n_{0} L^{3}>1$. This unphysical behavior is only possible if irreducible long-range multi-particle interactions, which can not be reduced to the effective chemical potential shift, are present in the system - something that is not observed in Nature.

The other problem with the Jastrow variational ansatz (and, of other such wave functions as well) is that it does not easily describe the physics of phase separation. Because, as shown above, a dilute Bose gas of vacancies in helium has attractive interactions and thus is an unstable, phase separating system, 
any discussion of BEC and superfluidity of the uniform vacancy gas under such circumstances is meaningless.

\section{$4{ }^{4} \mathrm{He}$ "ice cream"}

In this Section, we shall argue that many experimental facts suggest that the observed supersolid phenomenon in ${ }^{4} \mathrm{He}$ is induced by defects, or disorder, in the torsion oscillator samples. This point of view is supported by several numerical simulations, as well as by the recent direct observation of the grain boundary superflow [19].

Let us assume that the observed drop in the resonant period of the torsion oscillator can be attributed to a non-zero superfluid response at a given frequency. This seems plausible, as the period drop or, equivalently, mass decoupling increases as the oscillator amplitude $A_{o s c}$ and velocity $v_{\text {osc }}$ decrease. [To understand why $n_{s}$ decreases with $v_{\text {osc }}$, one has to consider the non-linear response of vortex loops and pinned vortex lines to the flow.] In normal viscous media, an opposite correlation between the mass decoupling and $v_{\text {osc }}$ is expected. One may also attempt to understand the NCRI effect in terms of kinetic relaxation or mass redistribution in the cell $[49,50]$. However, in helium samples, these explanations face the crucial blocked-channel test [49] — only superflow can be sensitive to the sample topology on macroscopic length-scales. It is also hard to believe that mass redistribution kinetics in solid helium is reversible at low temperature, i.e. the signal would not be reproducible upon heating and cooling (assuming that $T<1 \mathrm{~K}$ in the cycle to avoid possible annealing effects).

With the superfluid response in mind, we observe that many features in the data simply do not fit into the homogeneous supersolid picture. In particular:

\section{Dependence of the superfluid density on temperature}

It is a well established fact that in a continuous normal-superfluid (N-SF) transition, the superfluid density dependence on temperature near the transition point at $T_{\mathrm{c}}$ is given by $\left(T-T_{\mathrm{c}}\right)^{\nu}$, where $\nu \approx 0.671(1)$ is the correlation length exponent for the 3D XY-universality class [51]. So far, no exception to this law, which predicts an infinite derivative $d n_{s} / d T$ at $T_{\mathrm{c}}$ has been found, either theoretically or experimentally, and the same is expected for the transition to the SFS state [45]. On the other hand, in all torsional oscillator experiments displaying mass decoupling at low $T$, the rise of $n_{s}(T)$ at low $T$ starts off with zero derivative near $T_{\mathrm{c}}$, in turn rendering a precise determination of $T_{\mathrm{c}}$ rather ambiguous. The most straightforward explanation for such a gradual increase of the superfluid density would be a broad distribution of local transition temperatures $T_{\mathrm{c}}(\boldsymbol{r})$ within a strongly inhomogeneous sample. The shape 
of the $n_{s}(T)$ curve is then controlled by the probability distribution of transition temperatures. Diffraction experiments might provide information on the crystal quality in experimental samples but these were not reported yet.

Specific heat anomaly

The other puzzling feature, which does not conform to the established picture of the continuous N-SF transition, is the absence of the specific heat maximum. Given experimental uncertainties [3], the possible amplitude of the specific heat anomaly is orders of magnitude too small for the observed amount of the superfluid density [52]. Moreover, it was found that the dependence of the specific heat $C_{v}$ on $T$ is linear in the supersolid regime. A linear $C_{v}(T)$ may originate from one-dimensional Luttinger-liquid type structures, e.g., superfluid dislocation cores [52] and ridges between grain boundaries [26,46], or just from a collection of two-level systems in the amorphous sample [53].

\section{Dependence on geometry}

If the supersolid phenomenon observed in recent experiments is indeed a homogeneous bulk effect, it is difficult to explain the observed dependence of the microscopic ${ }^{1}$ parameter $n_{s}$ on the geometry of the experimental setup . The scatter of the reported values of $n_{s}$ (spanning almost a decade), depending on annulus or open geometry in the $\mathrm{KC}$ experiment, can be in principle explained if the sample quality, and thus the distribution of $T_{\mathrm{c}}$, is strongly dependent on the cell geometry (certainly a plausible argument).

\section{Dependence on sample "history"}

Rittner and Reppy [5] have reported the "elimination of supersolid by annealing", in an experimental setup very close to that of Kim and Chan. The other important observation, is that the torsional oscillator quality factor, or inverse dissipation rate, is higher in normal samples. This result should be regarded as direct evidence in favor of disorder-induced supersolidity. However, such annealing effects have not been confirmed by Kim and Chan [4], and the explanation for this discrepancy is lacking, at the time of this writing.

\section{${ }^{3}$ He effect}

Bosonic superfluidity is a very robust phenomenon, and one does not expect any significant changes in the superfluid properties when ${ }^{3} \mathrm{He}$ impurities are added, at a concentration $n_{3}<<n_{s}$. It is well established [54-56] that ${ }^{3} \mathrm{He}$ substitution atom in the ${ }^{4} \mathrm{He}$ solid matrix is described by a tight-binding model, with a tiny hopping amplitude $J \sim 10^{-4} \mathrm{~K}$. The small value of $J$ is consistent

\footnotetext{
${ }^{1}$ Microscopic is defined here as occurring on length scales much larger than the correlation length but much smaller than the system size
} 
with the characteristic energy for nuclear magnetism in solid ${ }^{3} \mathrm{He}$, which is believed to be due to exchange of helium atoms [57]. This result is important in several ways. First, it proves that ${ }^{3} \mathrm{He}$ atoms do not induce vacancy formation in their vicinity since the ${ }^{3} \mathrm{He}$-vacancy complex would be extremely mobile (the vacancy hopping amplitude is nearly four orders of magnitude higher [36]). Second, a small value of $J$ makes ${ }^{3} \mathrm{He}$ atoms extremely sensitive to virtually any type of crystalline disorder, since defects will certainly create tight bound states for ${ }^{3} \mathrm{He}$. Even a weak deformation potential between ${ }^{3} \mathrm{He}$ impurities is sufficient to bind them [54,58], or localize dilute ${ }^{3} \mathrm{He}$ solutions [59].

It is therefore very surprising, that both the superfluid fraction and the onset of the superfluid response observed in $\mathrm{KC}$ experiments, are significantly altered when ${ }^{3} \mathrm{He}$ impurities are added, even if the concentration $n_{3}$ is as small as few $p p m$, (or even few $p p b$ !). The mismatch between $n_{s}$ and $n_{3}$ is so large that it casts serious doubts on the homogeneous supersolid scenario [26], even on phenomenological grounds [60].

In a disordered sample, the lighter ${ }^{3} \mathrm{He}$ atom will try to minimize its kinetic energy by binding to static defects which have lower local particle number density, such as dislocation cores, grain boundaries and ridges between them (vacancies are mobile and it is not clear whether they can form a bound state with ${ }^{3} \mathrm{He}$ atoms). One thus expects an accumulation of ${ }^{3} \mathrm{He}$ on defects at low temperature; it is plausible that the local concentration of isotopic impurities may be fairly high in certain regions of space. The other, somewhat speculative, effect of ${ }^{3} \mathrm{He}$ substitution might be that the quality of samples, i.e. the amount of disorder, is itself a function of $n_{3}$. For example, by concentrating at the perimeter of the microcrystal, ${ }^{3} \mathrm{He}$ atoms may inhibit fast crystal growth, thereby helping to make the "ice cream".

If we now couple these considerations with the theoretical conclusion that only disorder can be responsible for the supersolid phenomenon in helium, we find a very subtle interplay between the superfluid response and ${ }^{3} \mathrm{He}$ content. Imagine for a moment, that superfluidity occurs along grain boundaries, while ${ }^{3} \mathrm{He}$ is concentrated at the boundary ridges. Assuming that ${ }^{3} \mathrm{He}$ atoms suppress superfluidity, we arrive at the picture where ${ }^{3} \mathrm{He}$-rich ridges form a Josephson junction network completely surrounding grain boundaries. In this scenario it is conceivable that even a tiny amount of ${ }^{3} \mathrm{He}$ (per volume) might be relevant for supersolidity [26].

\section{Dependence on pressure}

The obvious expectation is that supersolid properties ought to be suppressed, as pressure is applied to the sample. Applying pressure indeed drives down the superfluid transition temperature $T_{\mathrm{c}}$. Practically all known properties of helium crystals are consistent with the overall tendency of helium atoms to behave more classically at higher densities (see, e.g. Fig. 3). Surprisingly, the KC 
data [4] show hardly any pressure dependence for $n_{s}$ and $T_{c}$, up to $P \approx 65$ bar $^{1}$; at higher pressures, the supersolid signal weakens, and apparently disappears at $P>160$ bar. At this point one may wonder whether experimental data on pressure dependence should be attributed to properties of the same sample, as if the pressure was changed by squeezing the volume, or different point represents properties of different samples. If supersolidity is directly related to the sample quality, which, in turn, depends on initial conditions and the solidification protocol, then data become very ambiguous, and their interpretation intricate.

While the above mentioned experimental facts do not fit into the homogeneous supersolid crystal picture, there are others which are hard to reconcile with any existing theoretical framework. For example:

"Critical velocity"

Superfluid decoupling in the torsion bob has the most unusual dependence on the oscillator amplitude/velocity. Though in the current setup velocity and amplitude are strictly related, it is assumed that forces equilibrate in the sample fast enough to attribute all changes in the superflow to the velocity $v$. It is found that $n_{s}$ saturates at macroscopically small velocities, $v_{\text {sat }} \sim 10 \mu \mathrm{m} / \mathrm{s} \sim \hbar / \mathrm{mL}$ (related to just few circulation quanta). These values clearly have nothing to do with the naive sound velocity estimate $c \sim \sqrt{n_{s} U / m} \sim 10 \mathrm{~m} / \mathrm{s}$ based on typical helium parameters. The discrepancy is so large, that there is virtually no room left for explaining $v_{\text {sat }}$ using microscopic mechanisms. On the other hand, if extended defects in the superfluid phase order are involved, e.g. vortex lines of length $L$, then one faces the problem that the vortex motion timescale $m L^{2} / \hbar^{2}$ is 5 to 6 orders of magnitude too long, when compared to the oscillation period.

\section{Zero-flow experiments}

Superfluidity is associated with an anomalous mass current response to a gauge phase gradient or chemical potential difference. There have been numerous attempts in the past to detect superflow in the solid phase with negative results (see Ref. [17]). The same conclusion was reached in more recent studies [18], both in Vycor and in bulk samples. As they stand now, flow experiments do not support, and indeed are incompatible with the supersolid phase of helium. We can only note here that in disordered solids large pressure gradients are common, and it is not obvious what chemical potential difference is applied to the superfluid component in the disordered sample. For example, air pressure in the cave deep under the mountain is still at a modest 1 bar value.

${ }^{1}$ It should be mentioned that experimental data published so far are rather noisy 


\section{$4.1 \quad$ Superglass}

Aside looking at defects that are typical for the polycrystalline sample, one may wonder if helium can exist in an "ultimate" disordered solid phase, namely, a glass. All known structural glasses are in a normal, non-superfluid state. In this regard, ${ }^{4} \mathrm{He}$ offers an intriguing possibility, unique for a quantum solid, of being in a "superfluid glassy" phase, or superglass (SG). By definition, in the metastable SG phase the translation invariance is broken, but the pair correlation function $g(\boldsymbol{r})=\langle n(\boldsymbol{r}) n(0)\rangle$ features no diagonal long range order. At the same time, a superglass has ODLRO and non-zero superfluid response. Strange as it is, the SG is reminiscent of a sponge soaked in a superfluid liquid made of the same atoms.

Superglass was observed in numerical simulations of high-pressure (about 150 bar) samples prepared by fast temperature quench from the normal liquid state [32]. Clearly, the dynamics of real helium under cooling is vastly different from the thermalization dynamics in the Monte Carlo simulation done (i) in imaginary time, and (ii) in the absence of energy conservation. Thus, numerical simulations rather answer the question of existence of the metastable phase and its quasi-equilibrium properties, but not how easily it can be prepared experimentally. One may also wonder if helium SG is merely an artifact of the simulation algorithm (though we do not see any obvious reason to suspect that), or a long-lived, metastable, physical phase responsible for the unexpected outcome of the acoustic-pulse experiment [61] which aimed at nucleating the crystal phase in the middle of the cold, $50 \mathrm{mK}$, superfluid liquid but failed. The local pressure in the pulse reached 160 bar, well above the theoretically predicted threshold of 60 bar for quantum nucleation [62]. It is not known what the normal-superfluid transition temperature in the glassy phase is, at what temperature the metastable liquid freezes into a glass, and what role (if any) the SG might play in the torsion oscillator experiments, e.g., by being trapped in some amount between micro-crystals.

\section{Grain boundary superfluidity}

Even if the bulk phase of the material is an insulating solid, there is no physical reason why grain boundaries (GB) in the same material ought to be insulating as well, especially in the vicinity of the (weakly first-order) liquid-solid transition. It means that SF in lower dimensionality can be obtained by simply placing two solid pieces right next to each other. Conceptually, this possibility was demonstrated in model simulations of domain walls in the checkerboard solid formed by hard core bosons with the nearest-neighbor repulsion on a simple cubic lattice [63]. Subsequent path-integral Monte Carlo simulations of ${ }^{4} \mathrm{He}$ polycrystals [46] did reveal that some (not all !) grain boundaries are likely 

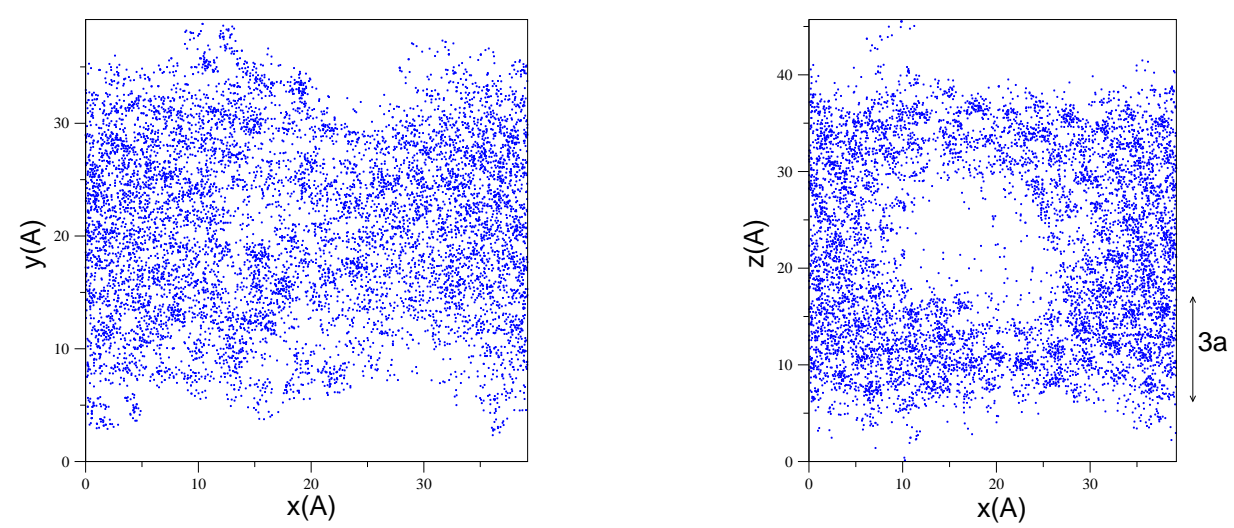

Figure 4. Phase coherence properties of grain boundaries and ridges in the ${ }^{4} \mathrm{He}$ sample consisting of two microcrystals with about $12 \times 12 \times 7$ atoms each. Periodic boundary conditions are assumed. The XY boundaries between different crystals were created by rotating both of them at random. Other grain boundaries are induced by periodic boundary conditions in the simulation box of rectangular shape, i.e. they are created within the same crystal. We forced YZ boundaries to be insulating by fixing atom positions in the YZ-layer ( $1.5 \mathrm{a}$ thick) at the equilibrium hcp points. Superfludity was then possible only along the $\hat{x}$ and $\hat{z}$ directions. Points in the figure are show positions of particles which participated in macroscopic exchange cycles with non-zero winding numbers, i.e. directly contributed to the superfluid response. The two panels (from left to right) projections on the XY (only the upper half of the sample is projected on the XY plane) and XZ planes. The Monte Carlo simulation was performed in the grand canonical ensemble at the coexistence curve.

to support superfluidity. Superfluidity in the layer at the disordered Vycor substrate was also reported in Ref. [64].

Simulations also find that nearly all ridges (lines of contact between different GB) show robust phase coherence properties. It is thus possible that superfluidity across grain boundaries of small (216-atom) crystals is nothing but the proximity effect. This issue was addressed in simulations of much larger GB [46] in a system of about 2000 atoms consisting of two randomly oriented crystallites at the melting density $n=0.0287 \mathrm{~A}^{-3}$. The main conclusions did not change; while grain boundaries at special angles and high symmetry directions are insulating, generic grain boundaries appear to be superfluid with typical transition temperatures (orientation dependent) of about half a Kelvin and the maximum possible $T_{c}$ at about $1.5 \mathrm{~K}$. The width of the superfluid GB region is about $\sim 3 a$ where $a$ is the nearest-neighbor distance in the hcp crystal, see Fig. 4.

In a remarkable recent experiment [19], superflow along the grain boundary was detected in an experiment whose setup is similar to that shown in Fig. 1. The difference between the solid levels was found to decay according to the linear law $\dot{h}=$ const characteristic of the superfluid flow at the critical velocity (estimated to be of the order of meters per second). The flow was detected only in the presence of grain boundaries; otherwise the sample did not relax. On one 
occasion, the dynamics abruptly came to a halt, as the boundary had suddenly disappeared. A grain boundary is a topological crystalline defect, and its quick (in less than 1 min time) disappearance is by itself an amazing observation, since it has no obvious explanation other than suggesting that boundaries are in a rough superfluid phase, supporting coherent recrystallization waves.

\section{Perspectives for future theoretical and experimental work}

It is currently our strong belief, that all known experimental facts, together with results of first-principles numerical simulations, rule out any explanation of the observed supersolid phenomenon in ${ }^{4} \mathrm{He}$ within a homogeneous crystal framework. The only reasonable alternative, consistent with many observations, is that of supersolidity induced by crystalline disorder. At the moment, very little is known for sure about structure properties of solid samples in the torsion oscillator experiments. Hopefully, in future investigations it will be possible to control sample quality in situ, e.g. by forming solids under constant pressure, rather than under constant volume conditions. Helium crystals of high quality are usually made out of the superfluid liquid at constant pressure. Solids grown from normal liquid under constant volume conditions are not transparent (similar in appearance to milk) [65]. It is also desirable to have direct information on the solid order in the same supersolid sample, e.g., by optical means. Even more urgently, several experimental observations of different groups have to be reconciled, especially regarding pressure dependence and annealing effects. It seems also that additional measurements of the specific heat and other thermodynamic properties can help in quantifying the amount of "disorder" in experimental samples [52].

Analytic theories of the SFS state in continuous space are phenomenological in nature when it comes to predicting, for a particular Hamiltonian, whether this state exists and what are crucial parameters for the SFS state in terms of bare couplings. Supersolids arise from competition between the kinetic and potential energy terms invalidating perturbative and mean-field treatments. At present, we do not know a single example of a system characterized by a realistic interatomic potential, which has a stable SFS phase in continuous space. This work is worth pursuing, since there are exciting new possibilities in engineering interparticle interactions in cold atomic systems. It would be also interesting to see, once the ideal SFS phase is found, which of the recent theoretical predictions capture the microscopic picture of the supersolid phase more adequately.

So far, the only observation of the superfluid glass phase was numerical (at $T=0.2 \mathrm{~K}$ and $n=0.0359 \mathrm{~A}^{-3}$ ). Most properties of the helium glass phase, and even its very existence, are a mystery. Theoretically, more work has to be 


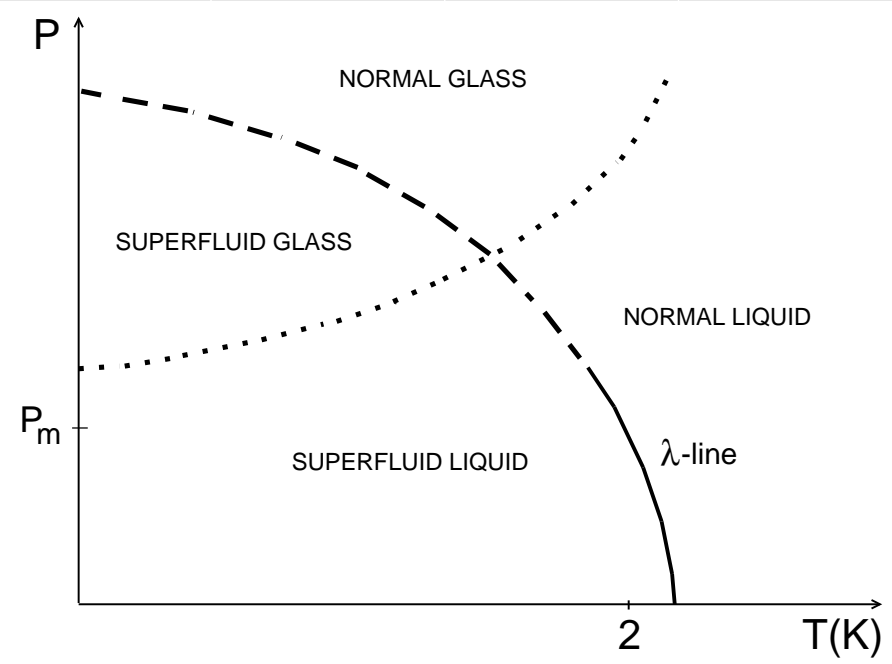

Figure 5. A sketch of the metastable helium glass phase diagram. Apart from the melting pressure $P_{m}$ we do not show the equilibrium liquid-solid line in the figure; the metastable glass state is supposed to be inside the stable solid phase. Since the liquid-solid transition is first-order the normal-superfluid transition in the metastable liquid is a continuation of the $\lambda$-line. The dashed line is indicating a sharp crossover, not the genuine phase transition; above this line the structural relaxation time is beyond the experimental reach.

done to quantify the SG state, and to see whether the heuristic phase diagram shown in Fig. 5 takes place. Experimentally, one may think of preparing glassy solid samples by fast density increase at low temperature rather then temperature quench at high density. Experimentally, it seems that in helium pressure increase can be achieved on a much shorter time scale than cooling down to $T=0.2 \mathrm{~K}$. [One objection to this proposal is that crystal growth from the cell wall at low temperature is extremely fast [66].] Another way of looking for non-ergodic properties of helium samples, i.e. system dependence on the intial conditions and preparation protocol, is to compare samples cooled while rotating with samples rotated after cooling [67].

At the moment, the grain boundary flow experiments give us the most unambiguous clue to the solution of the supersolid puzzle. On the other hand, a whole plethora of new questions arise in this connection. The problem is very broad, not only because crystalline interfaces can be prepared at different angles with respect to the crystal axes, but also because one can get different results, depending on how well the initial state was "annealed" (with respect to minimizing the classical deformation energy), and whether defects are "doped" with out-of-equilibrium vacancies and/or interstitials (one may wonder if vacancies cluster on defects the same way they do it in the bulk and whether they can make, say a dislocation core, superfluid ?). 
In the experimental setup of Ref. [19], the superflow in the boundary layer was observed only at the coexistence curve. It is important to extend this type of studies to higher pressures, though the experimental design has to be modified. One possibility [68], is to look at the same topology as in Fig. 1 with the bulk solid being sandwiched between two Vycor plates. Since helium in Vycor remains superfluid at pressures about 10 bar higher than the bulk melting value $P_{m}$ one may think of measuring the flow through the solid in response to the pressure difference between the two arms of the U-tube.

Another fascinating subject is recrystallization waves in the boundary layer. Though possible in principle [63] and being a logical explanation for the fast disappearance of grain boundaries in the experiment [19], recrystallization waves still remain to be seen and detected. Formally, there is no one-to-one connection between recrystallization waves and superfluidity in the boundary, e.g. smooth domain walls in the $3 \mathrm{D}$ checkerboard solid remain superfluid at zero-temperature, but the theory of the interplay between the two phenomena is basically non-existent.

Previous NMR studies of ${ }^{3} \mathrm{He}$ solid solutions [54-56,59] provided detailed information on the isotopic impurity hopping dynamics, as well as on interparticle interactions. These studies were performed at relatively high temperatures $T>0.7 \mathrm{~K}$ and in high quality crystals. It would be interesting to see if NMR techniques can be used to understand what happens to ${ }^{3} \mathrm{He}$ atoms in disordered samples grown from the normal high-density liquid and cooled down to the $100 \mathrm{mK}$ range. If ${ }^{3} \mathrm{He}$ atoms form a dense solution at the defects, one should be able to see it in the increased NMR relaxation rates since coupling between the ${ }^{3} \mathrm{He}$ magnetic moments is the only available mechanism. Another exciting possibility is to use NMR techniques to see if ${ }^{3} \mathrm{He}$ solids can be in the metastable glass phase.

The anomalously low critical velocity in the torsional oscillator studies is yet to be understood, even at the expense of admitting that the observed phase is, in fact, not superfluid [40].

\section{Concluding remarks}

Supersolids may happen in two ways: either as ideal incommensurate crystals with zero-point vacancies or commensurate crystals full of topological defects. Though the original observation of supersolidity in ${ }^{4} \mathrm{He}$ can not be interpreted in terms of zero-point vacancy mechanism in a perfect crystal, things turn out to be far more interesting and exciting than expected. Instead of one well-characterized crystal, we now face a whole variety of disordered solid samples. This immediately brings into focus grain boundaries, dislocations, and glassy phases which are, on their own right, new superfluid physical 
systems. For example, dislocations and grain boundaries should be considered as special low-dimensional objects inseparable from their three-dimensional environment. Studies of their fascinating properties make the experimental and theoretical landscape multi-dimensional, since in addition to pressure and temperature, the set of important parameters also includes various orientation angles. There is little doubt that this research field is going to last. We also believe that superfluid amorphous helium is a unique and remarkable state which will add a new angle to studies of glassy systems. Apart from understanding the role of crystalline defects in the supersolid phenomenon, it is extremely important to formulate what type of realistic interaction potential between particles can lead to the ideal supersolid phase - this long-term goal which started more than 50 years ago is yet to be achieved. Existing experimental and theoretical tools are more than capable of making significant progress in achieving these goals, both at the phenomenological and microscopic levels.

\section{Acknowledgment}

I am indebted to B. Svistunov, M. Boninsegni and M. Troyer for numerous discussions and valuable suggestions which lead to this work. I acknowledge financial support by the National Science Foundation under Grant No. PHY0426881.

\section{References}

[1] E. Kim and M. H. W. Chan, Nature 427, 225-227 (2004).

[2] E. Kim and M. H. W. Chan, Science 305, 1941-1944 (2004).

[3] A.C. Clark and M. H. W. Chan, J. Low Temp. Phys. 138, 853-858 (2005).

[4] E. Kim and M. H. W. Chan, Phys. Rev. Lett. 97, 115302 (2006).

[5] A. S. Rittner and J. D. Reppy, cond-mat/0604528.

[6] M. Kondo, S. Takada, Y. Shibayama, and K. Shirahama, cond-mat/0607032.

[7] A. Penzyev, and M. Kubota, unpublished, preliminary results are available online at http://online.kitp.ucsb.edu/online/smatter_m06

[8] A. J. Leggett, Phys. Rev. Lett. 25, 1543 (1970).

[9] O. Penrose, Phil. Mag. 42, 1373 (1951).

[10] O. Penrose and L. Onsager, Phys. Rev. 104, 576 (1956).

[11] C. N. Yang, Rev. Mod. Phys. 34, 694 (1962).

[12] A. J. Leggett, Science 305, 1921 (2004).

[13] A. F. Andreev and I. M. Lifshitz, Sov. Phys. JETP 29, 1107 (1969);

[14] G. V. Chester, Phys. Rev. A 2, 256 (1970).

[15] P. Sengupta, L.P. Pryadko, F. Alet, M. Troyer, and G. Schmidal, Phys. Rev. Lett. 94, 207202 (2005); G.G. Batrouni, F. Hebert, and R.T. Scalettar, Phys. Rev. Lett. 97, 087209 (2006).

[16] S. Wessel and M. Troyer, Phys. Rev. Lett. 95, 127205 (2005); D. Heidarian and K. Damle, Phys. Rev. Lett. 95, 127206 (2005); R. G. Melko, A. Paramekanti, A. A. Burkov, A. Vishwanath, D. N. Sheng, and L. Balents, Phys. Rev. Lett. 95, 127207 (2005); M. Boninsegni and N. Prokof'ev, Phys. Rev. Lett. 95, 237204 (2005).

[17] M. W. Meisel, Physica B: Cond. Matt. 178, 121 (1992).

[18] J. Day, T. Herman, and J. Beamish, Phys. Rev. Lett. 95, 035301 (2005); J. Day and J. Beamish, Phys. Rev. Lett. 96, 105304 (2006).

[19] S. Sasaki, R. Ishiguro, F. Caupin, H. J. Maris, and S. Balibar, Science 313, 1098 (2006). 
[20] W. Kohn, Phys. Rev. 133, A171 (1964).

[21] W. Kohn and D. Sherrington, Rev. Mod. Phys. 42, 1 (1970).

[22] V. L. Berezinskii, Sov. Phys. JETP 32, 493 (1970); ibid 34, 610 (1971); J. M. Kosterlitz and D. J. Thouless, J. Phys. C 6, 1181 (1973); J. M. Kosterlitz, J. Phys. C 7, 1046 (1974).

[23] H. R. Glyde, R. T. Azuah and W. G. Stirling, Phys. Rev. B 62, 14337 (2000).

[24] D. M. Ceperley, Rev. Mod. Phys. 67, 279 (1995).

[25] S. Moroni and M. Boninsegni, J. Low Temp. Phys. 136, 129 (2004).

[26] N. Prokof'ev and B. Svistunov, Phys. Rev. Lett. 94, 155302 (2005).

[27] L. Reatto Phys. Rev. 183, 334 (1969)

[28] R. A. Aziz, V. P. S. Nain, J. S. Carley, W. L. Taylor and G. T. McConville, J. Chem. Phys. 70, 4330 (1979).

[29] R. P. Feynman, Phys. Rev. 901116 (1953); ibid 911291 (1953).

[30] D. M. Ceperley and B. Bernu, Phys. Rev. Lett. 93, 155303 (2004).

[31] E. L. Pollock and D. M. Ceperley, Phys. Rev. B 36, 8343 (1987).

[32] M. Boninsegni, N. V. Prokof'ev and B. V. Svistunov, Phys. Rev. Lett. 96, 105301 (2006).

[33] B. K. Clark and D. M. Ceperley, Phys. Rev. Lett. 96, 105302 (2006).

[34] B. A. Fraass, P. R. Granfors and R. O. Simmons, Phys. Rev. B 39, 124 (1989).

[35] I. A. Todoshchenko, H. Alles, H. J. Junes, A. Ya. Parshin, V. Tsepelin, cond-mat/0607081.

[36] D. E. Galli and L. Reatto, Phys. Rev. Lett. 90, 175301 (2003).

[37] F. P. Lipschultz, V. J. Minkiewicz, T. A. Kitchens, G. Shirane, and R. Nathans, Phys. Rev. Lett. 19, 1307 (1967)

[38] M. Boninsegni, A. Kuklov, L. Pollet, N. Prokof'ev, B. Svistunov, and M. Troyer, Phys. Rev. Lett. 97, 080401 (2006)

[39] X. Dai, M. Ma, and F.-C. Zhang, Phys. Rev. B 72, 132504 (2005).

[40] P. W. Anderson, W. F. Brinkman and D. A. Huse, Science 310, 1164 (2005).

[41] M. Tiwari and A. Datta, cond-mat/0406124.

[42] G. Baskaran, cond-mat/0505160.

[43] Hui Zhai and Yong-Shi Wu, J. Stat. Mech. P07003 (2005).

[44] J. Ye, Phys. Rev. Lett. 97, 125302 (2006).

[45] A.T. Dorsey, P.M. Goldbart, and J. Toner, Phys. Rev. Lett. 96, 055301 (2006).

[46] L. Pollet, M. Boninsegni, A. Kuklov, N. Prokof'ev, B. Svistunov, and M. Troyer, in preparation. Preliminary results on superfluidity of grain boundaries in ${ }^{4} \mathrm{He}$ were reported at the KITP Miniprogram: The Supersolid State of Matter, February 6-17, (2006); [http : //online.kitp.ucsb.edu/online/smatter ${ }_{m} 06 /$ svistunov].

[47] S. Vitiello, K. Runge, and M. H. Kalos, Phys. Rev. Lett. 60, 1970 (1988).

[48] D.E. Galli, M. Rossi, and L. Reatto, Phys. Rev. B 71, 140506(R) (2005).

[49] A.C. Clark, X. Lin, M.H.W. Chan, cond-mat/0610240.

[50] Z. Nussinov, A.V. Balatsky, M.J. Graf, S.A. Trugman, cond-mat/0610743.

[51] J. A. Lipa, J. A. Nissen, D. A. Stricker, D. R. Swanson, and T. C. P. Chui, Phys. Rev. B 68, $174518(2003)$.

[52] A.V. Balatsky, M.J. Graf, Z. Nussinov, and S.A. Trugman, cond-mat/0606203.

[53] W. A. Phillips, J. Low Temp. Phys. 7, 351 (1972); P. W. Anderson, B. I. Halperin, and C. M. Varma, Phil. Mag. 25, 1 (1972).

[54] M.G. Richards, J.H. Smith, P.S. Tofts, and W.J. Mullin, Phys. Rev. Lett. 34, 1545 (1975).

[55] M. G. Richards, J. Pope, P. S. Tofts, and J. H. Smith, J. Low. Temp. Phys. 24, 1 (1976); A. R. Allen, M. G. Richards, J. Schratter, J. Low. Temp. Phys. 47, 289 (1982).

[56] V. A. Mikheev, V. A. Maydanov, and N. P. Mikhin, Solid State Comm. 48, 361 (1983).

[57] R.A. Guyer, R.C. Richardson, and L.I. Zane, Rev. Mod. Phys. 43, 532-600 (1971).

[58] A. F. Andreev, Sov.Phys. - JETP 41, 1170 (1976).

[59] Yu. Kagan and L.A. Maksimov, Sov. Phys. - JETP 60, 201 (1984).

[60] A. V. Balatsky and E. Abrahams, cond-mat/060253.

[61] F. Werner, G. Beaume, A. Hobeika, S. Nascimbene, C. Herrmann, F. Caupin and S. Balibar, J. Low Temp. Phys. 136, 93 (2004).

[62] F. Caupin, S. Balibar, and H. J. Maris, Physica B 329-333, 356 (2003).

[63] E. Burovski, E. Kozik, A. Kuklov, N. Prokof'ev, and B. Svistunov, Phys. Rev. Lett. 94, 165301 (2005).

[64] S.A. Khairallah and D.M. Ceperley, Phys. Rev. Lett. 95, 185301 (2005).

[65] R. Hallock, private communication.

[66] S. Balibar, T. Mizusaki, and Y. Sasaki, J. Low Temp. Phys. 120, 293 (2000); X. Chavanne, S. Balibar, and F. Caupin, Phys. Rev. Lett. 86, 5506 (2001); J. Low Temp. Phys. 125155 (2001). 
[67] M. Kubota, private communication.

68] R. Hallock and M. Ray, in preparation.

[69] H. P. Büchler, E. Demler, M. Lukin, A. Micheli, N. Prokofiev, G. Pupillo, and P. Zoller, Strongly Correlated 2D Quantum Phases with Cold Polar Molecules: Controlling the Shape of the Interaction Potential, cond-mat/0607294.

[70] T. Schneider and C.P. Enz, Theory of the Superfluid-Solid Transition of ${ }^{4} H e$, Phys. Rev. Lett. 27, 1186-1188 (1971).

[71] K. Goral, L. Santos, and M. Lewenstein, Quantum Phases of Dipolar Bosons in Optical Lattices, Phys. Rev. Lett. 88, 170406 (2002). 